\title{
Development Path and Urgency of Further Strengthening Construction of Public Hospitals Based on Novel Coronavirus Pneumonia Treatment
}

\author{
Jia-zhi LIAO, Jing WU \\ Tongji Hospital, Tongji Medical College, Huazhong University of Science and Technology, Wuhan 430030, China
}

(C) Huazhong University of Science and Technology 2020

\begin{abstract}
Summary: During the outbreak of novel coronavirus pneumonia (NCP) in Wuhan, public hospitals have played an important role in intensive care, case guidance and scheme optimization. At the same time, it also faces unprecedented challenges and tests. Based on the treatment of severe patients in Wuhan, combined with the treatment practice in Tongji Hospital affiliated to Tongji Medical College, Huazhong University of Science and Technology, this paper puts forward the urgency of further strengthening the construction of public hospitals, discusses the feasible path for promoting the development of public hospitals, so as to meet the growing medical needs of the people, improve the ability to respond to major public health emergencies, and effectively guarantee the safety of people's lives and the promotion of a healthy China construction.

Key words: novel coronavirus pneumonia; public hospital; epidemic prevention and control; public health emergency; construction
\end{abstract}

The first novel coronavirus pneumonia (NCP, also named COVID-19) that caused by novel coronavirus (later named SARS-CoV-2) was discovered in Wuhan, Hubei Province in December 8, 2019 ${ }^{[1]}$. The epidemic caused by COVID-19 gradually spread to the whole country and even the world. The situation is severe and changing rapidly. As of 24:00 on March 14, 2020, there are 80844 confirmed cases and 3199 deaths in China, with a mortality rate of $3.96 \%$. Among them, 49 999 cases have been confirmed in Wuhan City, where the epidemic started, 2456 people have died, with a mortality rate of $4.91 \%{ }^{[2]}$.

The NCP is the most serious public health emergency since New China was established. We should give scope to the role of a high-level medical team and play a better role in critical care, case guidance and scheme optimization ${ }^{[3]}$. President Xi Jinping stressed in the important speech on the coordination of the NCP prevention and control, economic and social development work conference in February 23, 2020. However, for Wuhan City and even the whole Hubei Province, the task of medical treatment is particularly heavy. Wuhan public hospital mobilizes all available medical resources and medical personnel, facing unprecedented challenges and tests, which not only reflects the responsibilities of public hospitals in the fight against the epidemic, but also exposes the gap in

Jia-zhi LIAO, E-mail: liaojiazhi@tjh.tjmu.edu.cn \#Corresponding author, E-mail: tjglzx@tjh.tjmu.edu.cn the allocation of space, personnel and resources.

To satisfy the increasing medical needs of the public ${ }^{[4]}$, and enhance the ability to cope with major public health emergencies ${ }^{[5-7]}$, this paper bases on the overall situation of NCP in Wuhan, and combines the experience of Tongji Hospital affiliated to Tongji Medical College of Huazhong University of Science and Technology in the fight against NCP, puts forward some suggestions for further strengthening the construction of public hospitals, hoping to ensure the safety of people's lives and promoting the construction of a healthy China.

\section{PUBLIC HOSPITALS: THE MAIN FORCE IN THE RESCUE WORK OF NCP}

Public hospitals are the main part of providing medical services, which may play an irreplaceable role in providing medical services for residents, training medical technical personnel, and safeguarding the health level of the people ${ }^{[8]}$. Since 2009, the state and relevant health departments have issued a series of documents, which have gradually clarified that public hospitals should undertake public health content such as disease prevention, control and emergency treatment, which conforms to the public welfare attribute of public hospitals, but in practice, the content and quality of public health services provided by public hospitals are uneven ${ }^{[9]}$.

Since the outbreak of NCP, the country has been 
fighting the epidemic with the whole nation to suppress the spread of the epidemic, and the public hospitals are the core force of medical services in Hubei Province and Wuhan City. In the process of epidemic prevention and control, more than 40 hospitals in Wuhan have been expropriated as NCP designated hospitals, most of them are public hospitals. The beds number for NCP patients increased from more than 5000 to 23 thousand in one month, of which 9000 beds were for severe patients. Sixteen mobile cabin hospitals have been built and opened with 13348 beds, which are taken over by public hospitals or military system medical personnel. It fully proved that the public hospitals are the main battlefields of the epidemic, and the national medical teams are mainly public medical and military medical personnel. Huazhong University of Science and Technology is the university that invests the most beds and medical staff in China. It has 10 affiliated hospitals (Tongji Hospital, Union Hospital, Liyuan Hospital, Puai Hospital, etc.), 8900 beds, 33000 medical staff devoted to fighting "epidemic" $[10]$, and has undertaken the rescue and treatment of severe patients. China's national system and the basic nature of medical services determine that the medical services of public hospitals are more accessible, higher quality and lower price. Especially in major public health emergencies, public hospitals respond quickly, medical staff are more subjected to deployment, and the emergency mechanism is easier to start ${ }^{[11]}$.

Therefore, taking the NCP as a guide, we must further strengthen the main position and main force role of public hospitals.

\section{THE URGENCY OF THE CONSTRUCTION AND DEVELOPMENT OF PUBLIC HOSPITALS}

Public hospitals in Hubei, especially in Wuhan, have been responsible for the diagnosis and treatment of NCP patients since the outbreak of the disease. However, NCP is unknown and refractory at first. In the face of NCP outbreak, the number of patients continues to increase, which affects the normal operation and management order of the hospital. Hospitals are facing increasing difficulties and pressures. The difficulties of bed resource shortage, equipment shortage, medical materials shortage and medical staff shortage are increasingly prominent. The NCP patients can't be implemented in time and in full, resulting in a high mortality rate in Hubei, especially in Wuhan. As of 24:00 on March 14, 2020, the mortality rate in Hubei Province is $4.55 \%$, that in Wuhan City is $4.91 \%$, and that outside Hubei Province is $0.87 \%$, which has attracted great attention of the central government ${ }^{[12]}$. More than 40000 medical and nursing personnel were sent to Hubei and Wuhan successively from 29 provinces, regions and cities, Xinjiang production and Construction Corps and military system ${ }^{[13]}$. The support of medical forces from other provinces plays an important role in preventing the epidemic. This incident reflects that it is urgent to strengthen the construction of public hospitals, promote the development of public hospitals, especially improve their ability to cope with major public health events.

According to the statistics center of the National Health Commission, by the end of 2018, there were 33009 hospitals in China, including 12032 public hospitals and 20977 private hospitals. Compared with 2017 , the number of hospitals increased by $1953^{[14]}$. Although the total number of hospitals continues to increase, however, the overall medical resources in China are still in an absolutely inadequate state. According to the latest data announced by the National Health Commission, in 2019, the number of doctors and nurses per thousand population reached 2.59 and 2.94 respectively ${ }^{[15]}$, still less than the national average of Organization for Economic Co-operation and Development (OECD) (3.4) ${ }^{[16]}$. There is huge demand for medical development. From 2005 to 2019, the number of private hospitals increased from more than 3200 to 20977 , doubling every two years on average, accounting for $63.5 \%$ of the total number of hospitals in China. In June 2019, the opinions on promoting the sustainable and healthy development of social medical institutions jointly issued by ten ministries and commissions stressed that "strictly control the number and scale of public hospitals, and leave enough space for the development of social medical institutions"[17]. But encouraging social medical institutions does not mean or need to restrict the development of public hospitals. The construction and development of public hospitals need the support of policy level.

\section{DEVELOPMENT PATH OF PUBLIC HOSPITALS REFLECTED BY EPIDEMIC SITUATION}

In the face of problems and deficiencies reflected in the treatment of the NCP epidemic, and to improve the response capacity of the public hospitals which are "main anti epidemic force" in public health emergencies, we put forward the following development suggestions. 3.1 Allocation of Equipment and Personnel for Public Hospitals in Line with Medical Needs in the New Era

In December 2018, the State Council's report on the distribution and use of financial medical and health funds pointed out that for public hospitals, the government is mainly responsible for the expenditure of capital construction and equipment purchase, development of key disciplines, personnel training, retirees' expenses and policy losses. The government, in accordance with the principle of "relying on the 
government for construction and relying on services for operation", grant subsidies to public hospitals in line with the regional health plan.

In fact, the proportion of government direct financial investment in the six major expenditures is not high in all parts of the country. From the second half of the 1990s, the proportion of government financial investment in all medical expenditures is only about $15 \%-18 \%{ }^{[18]}$. Public hospitals are more "relying on services for operation and on themselves for construction". At the same time, with the change of disease spectrum, the occurrence of public emergencies and the development of medical science and technology, the requirements of medical treatment for equipment, personnel and information technology are becoming higher and higher, while when the zero plus of hospital drug consumables, the service and technical income have not yet met the demand, resulting in the limited ability and space of hospital equipment and personnel, barely meeting the daily needs.

After the outbreak of NCP, although public hospitals in Wuhan actively vacate beds, there are obvious shortages of severe treatment facilities such as ventilators, ECMO and monitors. The shortage of medical staff, especially in respiratory, critical and emergency care, has become the biggest bottleneck, affecting the early diagnosis and treatment of patients. Relying on the Party Central Committee and the State Council to coordinate the medical personnel of the whole country to help, transfer or purchase equipment urgently. Meanwhile, learned from this novel coronavirus pneumonia, we recommended that the hospital should establish a complete emergency management system, develop a talent training plan based on the long-term development of emergency management, and build a professional team to deal with the possible public health event in the future ${ }^{[19]}$.

Only by implementing the public hospitals "construction depends on the government" and ensuring the allocation and operation funds of equipment and personnel, public hospitals can truly implement the "two permits", establish a salary system in line with the characteristics of the medical industry, allocate equipment and personnel more reasonably, better meet the daily medical needs, and more effectively respond to the unexpected needs of major public health events.

\subsection{Paying Attention to and Upgrade the Information Construction of Public Hospitals}

In July 2015, the State Council's guiding opinions on actively promoting the "Internet +" action clearly put forward the idea of enriching the application of internet in the fields of health care and other fields, so that the public can enjoy more fair, efficient, highquality and convenient services ${ }^{[20]}$. In April 2018, the general office of the State Council issued the opinions on promoting the development of "Internet + Medical
Health", encouraging medical institutions to use information technology such as the internet to expand the space and content of medical services ${ }^{[21]}$. The public hospitals have responded positively to the notifications of the National Health Commission on "strengthening the information technology to support the prevention and control of new coronavirus infections", and adopted the information support method, based on the medical cloud platform, to form a multi-district linkage epidemic prevention and management mode ${ }^{[22]}$. In the treatment of NCP patients, the application of information diagnosis and treatment system in public hospitals promotes the screen of NCP suspected cases and flexible treatment for non-NCP patients ${ }^{[23]}$. Take Tongji Hospital for example, on January 24, 2020, the online diagnosis and treatment of fever clinic was opened. The number of consultations on the first day was over 10000 . As of 24:00 on March 14, the total number of consultations was 84556 times. Tongji cloud clinic was opened in February 14, video diagnosis and treatment of non-NCP patients, cumulative diagnosis and treatment volume was 15202 people. Then nearly 200 hospitals across the country successively applied for the internet hospital online. However, information construction is still the necessary hidden cost for the modernization of public hospitals, and its investment can't reflect the value in the medical service charging system, resulting in obvious shortcomings in the information construction of public hospitals, which needs the support of national financial investment.

\subsection{Further Improving the Hierarchical Diagnosis and Treatment System, and Clarifying the Leading Position and Role of Public Hospitals}

On September 8, 2015, the general office of the State Council issued the guiding opinions on promoting the construction of hierarchical diagnosis and treatment system $^{[24]}$, which established the hierarchical diagnosis and treatment system of the first diagnosis at the grassroots level, two-way referral, rapid and slow treatment, and linkage between the upper and lower levels, which is planned to be basically realized during the "13th Five-Year Plan" period. Since the outbreak of NCP, the situation of medical treatment has made people realize the importance of strengthening the construction of large public hospitals, giving full play to the role of the leading hospitals of hierarchical diagnosis and treatment and its supporting function, which is important as strengthening the basic level, and may be more important to improve the success rate of severe (critical) treatment.

Taking Wuhan as an example, the peak rate of severe cases reached $31.6 \%$ in February, and the designated hospitals facing "medical squeeze" could not bear the task of centralized treatment, severe patients "waiting for bed", once the phenomenon of severe patients asking for help online appeared. It was 
not until Huoshenshan and Leishenshan hospitals are completed and put into use, the fourth and fifth batch of designated hospitals were transformed and put into use that the situation of "bed waiting for patients" gradually came into being. It has been proved that further increasing the investment in the construction of public hospitals, establishing an export mechanism for the urgent and critical diseases or public emergencies, and ensuring the smooth referral mechanism for the difficult, urgent and severe diseases have irreplaceable significance for the stable and efficient operation of the hierarchical diagnosis and treatment system.

\subsection{Public Hospitals Taking the Lead in Building National and Regional Medical Centers}

In February 2017, the National Health Commission launched the construction plan, planning to set up national medical centers according to comprehensive and different specialty categories nationwide by 2020 . The national medical center will play a leading role in the diagnosis and treatment of difficult and critical diseases, the training of high-level medical talents, and the research of high-level basic medicine. The national regional medical center will coordinate with the national medical center to promote the level of regional medical, prevention and health care services.

During the NCP epidemic prevention and control period, Tongji Hospital affiliated to Tongji Medical College of Huazhong University of Science and Technology, which is the leading hospital of Hubei Province declaration national regional medical center (comprehensive), extended the fever clinic in time, and actively rebuilt two new hospitals, and quickly started 2025 severe beds that meet the requirements of infectious diseases. As of March 14, 2020, 35979 fever outpatients (including emergency patients) were completed, 3228 severe patients were admitted, 1802 patients were cured and discharged, and 4191 medical staff participated in the first-line treatment, undertaking the largest number of available critical beds, the severe patients admitted, and the fever outpatient visits in a single hospital. Giving consideration to emergency treatment of non-NCP at the same time, the Tongji Hospital has been operating in the extreme state for a month. This suggests that the state needs to further increase investment in the comprehensive and specialized construction of national regional medical centers to improve their medical treatment level and clinical research capacity, and constantly strengthen the center's strength, so that it can't only play a leading role in daily diagnosis and treatment, radiation and talent training, but also play a leading role in major public emergencies, and play a mainstay effect.

\section{SUGGESTION AND CONSIDERATION}

"Under the strong leadership of the Party Central
Committee and through hard work, the current situation of epidemic prevention and control is developing in a good direction. The achievements of the prevention and control work once again demonstrate the remarkable advantages of the leadership of the Communist Party of China and the socialist system with Chinese characteristics." President Xi Jinping pointed out in his important speech in February 23rd. At the time of initial control of the epidemic situation, thinking about the long term, it is very important to establish the policy guidance of strengthening the construction and sustainable development of public hospitals from the perspective of national policies.

(1) It is very important to establish the layout and construction of national medical centers and regional medical centers as the guide, large-scale public hospitals as the leader, and constantly improve the construction of hierarchical diagnosis and treatment system at the strategic level.

(2) We should further improve the proportion of financial investment, scientifically verify the bed numbers, equipment allocation, personnel ratio, charging mode and salary standard of public hospitals, further strengthen the main position of public hospitals in the medical system at the right time, effectively meet the growing medical needs of the people, improve the ability to respond to major public health events, and make public hospitals truly become the defender of people's life safety and the main force of healthy China construction at planning level.

(3) Paying attention to the construction and development of special hospitals for infectious diseases under the condition of market economy at the financial security level, especially the establishment of special hospitals for infectious diseases in cities above the county level, so as to timely respond to the detection, isolation and treatment capacity of infectious diseases.

(4) Building an information-based early warning system at hospital prevention and control level, optimizing the pre inspection mode, moving forward to the appointment link, automatically collecting and analyzing the key words of epidemic situation in the process of diagnosis and treatment, conducting multi-dimensional comparison with infectious disease transmission elements, conducting intelligent evaluation, triggering early warning in time, and realizing the forward movement of prevention and control gateway.

\section{Conflict of Interest Statement}

The authors declare that they have no conflict of interest.

\section{REFERENCES}

1 Yang XY, Miu CL, Jin MD, et al. Current status and 
progress of 2019 novel coronavirus pneumonia. Chin Crit Care Med (Chinese), 2020,32(02): E006-E006.

2 Health and Health Commission of China. Novel coronavirus pneumonia epidemic situation up to 24 March 14th. (2020-03-01)[2020-03-01]. http://www. nhc.gov.cn/xcs/yqtb/202003/8331f126d3854413b6ea32 3009fbbcc5.shtml.

3 Xinhua Net. Xi Jinping's "2. 23" speech: The program of war "epidemic" aiming at total victory. (2020-02-25) [2020-03-01]. http://www.xinhuanet.com/politics/202002/25/c_1125625675.htm.

4 National Health and Health Commission of the People's Republic of China, State Administration of Traditional Medicine of China. Notice on issuing action plan for further improvement of medical services(2018-2020)(2018-01-04)[2020-03-01]. http://www.nhc.gov.cn/xxgk/pages/viewdocument. jsp?dispatchDate $=\&$ staticUrl $=/$ yzygj/s3594q/201801/9 df87fced4da47b0a9f8e1 ce9fbc7520.shtml\&wenhao.

5 Wang J, Yuan B, Li Z, et al. Evaluation of Public Health Emergency Management in China: A Systematic Review. Int J Environ Res Public Health, 2019,16(18):3478

6 Rose DA, Murthy S, Brooks J, et al. The Evolution of Public Health Emergency Management as a Field of Practice. Am J Public Health, 2017,107(S2):S126-S133

7 Martinez D, Talbert T, Romero-Steiner S, et al.Evolution of the public health preparedness and response capability standards to support public health emergency management practices and processes. Health Secur, 2019,17(6):430-438

8 Fang PS, Chen JY, Liu SL, et al. Challenges and consideration of public hospitals under the hierarchical diagnosis and treatment system. Chin Hosp Manag (Chinese), 2018,38(10):1-3

9 Meng YL, Qiu WQ, Wang K, et al. Analysis of disease prevention and control services provision at public tertiary general hospital in Beijing. Chin J Hosp Admin (Chinese), 2019,35(9):732-737

10 Tencent. Huazhong University of Science and Technology is the university that invests the most beds and medical staff in China, and 10 affiliated hospitals invest in epidemic war. (2020-02-21)[ 2020-03-01]. https://new. qq.com/omn/20200221/20200221A0TSQS00.html.

11 Zhou T. The prevention and contrition of novel coronavirus pneumonia manifest the advantage of China's medical system. Shanghai Economic Research (Chinese), 1-9[2020-03-01]. https://doi.org/10.19626/j. cnki.cn31-1163/f.20200220.001.

12 Fan DY. Try our best to further reduce the mortality of novel coronavirus pneumonia. Beijing Youth Daily, (2020-02-10) [2020-03-01]. https://3w.huanqiu.com/a/ $\mathrm{c} 4 \mathrm{~b} 13 \mathrm{~d} / 3 \mathrm{wyIj} 04 \mathrm{axhB}$ ? $\mathrm{p}=1$ \&agt $=8$.

13 Heroic city, heroic people. People's Daily. (2020-02-
25). https://baijiahao.baidu.com/s?id=16594823379185 $85606 \& w f r=$ spider $\&$ for $=$ pc.

14 Health and Health Commission of China. 2018 statistical bulletin of China's health development. (2019-05-22) [2020-03-01]. http://www.nhc.gov.cn/guihuaxxs/s1074 8/201905/9b8d52727cf346049de8acce25ffcbd0.shtml.

15 Health and Health Commission of China. The number of doctorsperthousand population in Chinareaches 2.59.(201909-26)[2020-03-01].http://news.cctv.com/2019/09/26/ ARTIWoF5GSg6C8JBZIBRsFaL190926.shtml.

16 Zan X, Zhu HP. The characteristics of American medical system and its enlightenment to China. Comparison, 2016, 6. http://www.casscppr.org/article/yiliaogaige/ other/1694.html.

17 Health and Health Commission of China. Notice on printing and distributing the opinions on promoting the development of sustainable health and standardization of social medical services. (2019-06-12)[2020-03-01]. http://www.nhc.gov.cn/yzygj/s3577/201906/8e83be9a9 9764ea6bb5f924ad9b1028d.shtml.

18 China Economic Weekly. The government's financial investment in public health should be increased to $3 \%$ of GDP. (2018-12-08)[2020-03-01]. http://paper.people. com.cn/zgjjzk/html/2008-12/08/content_154410.htm.

19 Qiu Y. Analysis on the current situation and countermeasures of emergency management of public health emergencies in hospitals. Quanke Kouqiang Yixue Zazhi (Chinese), 2019,6(36):12

20 State Council of China. Guidance on actively promoting the "internet +" action (State Office [2015] 40) (201807-1)[2020-03-01].http://www.gov.cn/zhengce/ content/2015-07/04/content_10002.htm.

21 State Council of China. Opinions on promoting the development of "internet + medical health" (GBF [2018] No. 26) (2018-04-28)[2020-03-01]. http://www. gov.cn/zhengce/content/2018-04/28/content_5286645. htm.

22 Ren YF, Zhang XX, Li J, et al. Practical exploration of the informationization support for prevention and control of the new coronavirus pneumonia with Tongji Hospital as an example. Chin J Hosp Admin (Chinese), 2020,36(00):E003-E003

23 Guo QF, Yang KQ, Zhao LT, et al. Novel coronavirus pneumonia prevention and control personnel management platform based on "internet + ". Nursing Res (Chinese), 1-3[2020-03-01]. http://kns.cnki.net/ kcms/detail/14.1272.R.20200218.1547.002.html.

24 State Council of China. Guidance on promoting the construction of hierarchical diagnosis and treatment system (GBF [2015] No. 70). (2015-09-8) [2020-0301].http:/www.gov.cn/zhengce/content/2015-09/11/ content 10158.htm.

(Received Mar. 10, 2020; revised Mar. 15, 2020) 